\title{
Selección de pozos candidatos a fracturamiento hidráulico en el campo Gustavo Galindo Velasco
}

\author{
Mauricio Adrián Bustos Moreno \\ Facultad de Ciencias de la Ingeniería - Carrera de Ingeniería en Petróleo. Universidad Estatal Península de Santa Elena \\ (UPSE). Avda. Principal La Libertad - Santa Elena. La Libertad. Ecuador. \\ Email:sc.sym@hotmail.com
}

\begin{abstract}
Resumen
El Campo Gustavo Galindo Velasco ubicado en la provincia de Santa Elena, ha comenzado a mostrar signos de envejecimiento o madurez, denominándose campo maduro debido a la reducción de la presión del yacimiento, lo que ocasiona una disminución en la producción de los pozos.

El fracturamiento hidráulico es una técnica de estimulación que permite obtener, mediante la creación de fracturas o canales que mejoran la interconexión de la permeabilidad en la formación, el aumento de la conductividad y el área de flujo de los fluidos hacia el pozo, incrementando su producción y prolongando la vida de los campos maduros.

En la actualidad, para emplear esta técnica de estimulación, es necesario realizar un análisis investigativo del historial de producción y reacondicionamiento efectuados en cada pozo, estimación de sus reservas, interpretación de registros eléctricos, calidad del cemento y diseño de fractura.

Los resultados obtenidos de la investigación, permiten aproximar el incremento de la producción después de efectuar el fracturamiento hidráulico en los pozos seleccionados y el análisis económico respectivo del proyecto, mediante el uso de los indicadores de rentabilidad.
\end{abstract}

Palabras claves: Fracturamiento Hidráulico, Estimulación, Tope del Cemento (TOC).

\section{Selection of candidate wells to hydraulic fracturing in the Gustavo Galindo Velasco field}

\begin{abstract}
The Campo Gustavo Galindo Velasco located in the province of Santa Elena, has begun to show signs of aging or maturity, being called mature field due to the reduction of the reservoir pressure, which causes a decrease in the production of the wells.

Hydraulic fracturing is a stimulation technique that allows obtaining, by means of the creation of fractures or channels that improve the interconnection of the permeability in the formation, increase of the conductivity and area of flow of the fluids towards the well, increasing its production and prolonging the life of mature fields.

Today, to employ this technique of stimulation, it is necessary to carry out an investigative analysis of the production history and reconditioning carried out in each well, estimation of their reserves, interpretation of electric logs, quality of the cement and design of fracture.
\end{abstract}

The results obtained of the research allow approximating the increase in production after performing the hydraulic fracturing in the selected wells and the respective economic analysis of the project, through the use of profitability indicators.

Keywords: Hydraulic Fracturing, Stimulation, Top of Cement (TOC)

Cita: Bustos, M.A. (2018). Selección de pozos candidatos a fracturamiento hidráulico en el campo Gustavo Galindo Velasco. Revista Fuentes: El reventón energético, 16(1), 81-86. 


\section{Introducción}

El Fracturamiento Hidráulico es una conocida técnica de estimulación de pozos (Quintero, Triana, Jaimes \& Torres, 2010). Con la finalidad de subir la producción de los pozos, desde los años 1950 se la utilizó en todas las áreas del Bloque Gustavo Galindo Velasco en las formaciones de los Grupos Azúcar y Ancón, obteniéndose muy buenos resultados.

Con estos antecedentes, la empresa Pacifpetrol S.A., operadora actual del Bloque Gustavo Galindo Velasco, en aras de seguir buscando oportunidades e incrementar el índice de productividad de los pozos, se planteó realizar punzados y estimulaciones (Fracturamiento Hidráulico) en las formaciones del Grupo Ancón. Este trabajo resume el proceso de selección del pozo ANC1968 candidato para punzamiento de intervalos adicionales y fracturamiento en una etapa.

\section{Antecedentes}

En la sección Tigre (zona central del campo) se encuentran perforados pozos relativamente profundos cuyo objetivo ha sido producir de las formaciones Atlanta (AT), Passage Beds (PB), Santo Tomás (ST), Clay Pebble Beds (CPB) y Socorro (SO). Los pozos cercanos al ANC1968 que han producido de estas formaciones presentan una producción acumulada promedio de 59.634 Bls (ver Tabla 1).

El valor de la producción acumulada de estos pozos se lo ha graficado en la Figura 1 donde se tiene un promedio de 59.634 Bls de petróleo de 4 pozos.

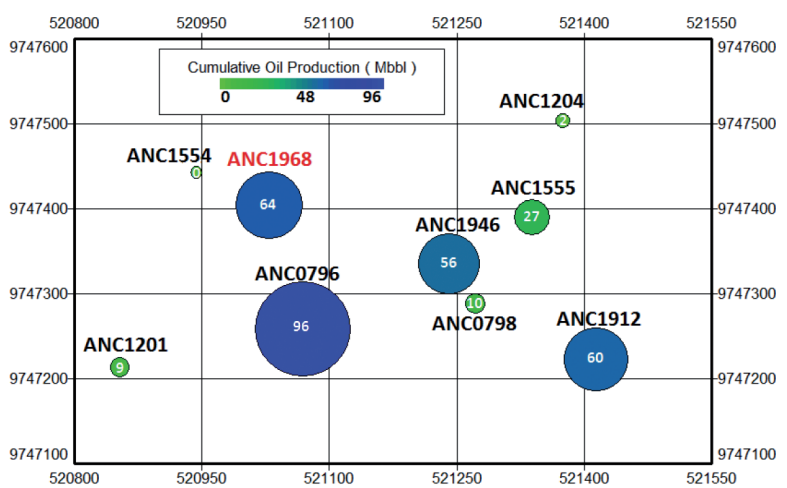

Figura 1. Producciones acumuladas de los pozos cercanos al ANC1968. Fuente: Pacifpetrol.

El pozo ANC1968 fue completado con casing de 41/2" hasta 4670 ' y abierto originalmente en las formaciones Atlanta y Santo Tomás. A la fecha tiene un acumulado de aprox. de $64 \mathrm{MBls}$ de petróleo.

En abril 1967 el pozo ANC1968 se punzó y fracturó en el intervalo 2013'-2209' de la parte inferior de la formación Santo Tomás incrementando su producción a $47 \mathrm{Bppd}$, sin embargo la parte superior de la formación Santo Tomás en el intervalo $1600^{\prime}-1720^{\prime}$ no fue punzada ni fracturada debido a que el tope de cemento se encontraba a 1960 '.

Es importante mencionar que en mayo del 2016 el pozo vecino ANC1912 fue punzado y fracturado en la parte superior de la formación Santo Tomás obteniéndose inicialmente 32 Bppd.

Tabla 1. Pozos productores de las formaciones AT-PB-ST-CPB-SO cercanos al ANC1968.

\begin{tabular}{ccccccccc} 
Pozo & $\begin{array}{c}\text { DT } \\
\text { (ft) }\end{array}$ & $\begin{array}{c}\text { Formaciones } \\
\text { Productoras }\end{array}$ & $\begin{array}{c}\text { Q inicial } \\
\text { (BPPD) }\end{array}$ & $\begin{array}{c}\text { Sistema de } \\
\text { Extracción }\end{array}$ & $\begin{array}{c}\text { Q Actual } \\
\text { (BPPD) }\end{array}$ & $\begin{array}{c}\text { Coordenada } \\
\text { X }\end{array}$ & $\begin{array}{c}\text { Coordenada } \\
\text { Y }\end{array}$ & $\begin{array}{c}\text { Petróleo Acumulado } \\
\text { (BLS) }\end{array}$ \\
\hline ANC0796 & 4489 & CPB/ST/AT & 30 & SW & 2,00 & 521067 & 9747260 & 96130 \\
ANC1946 & 3290 & AT & 50 & BM & 1,00 & 521239 & 9747337 & 55560 \\
ANC1555 & 1873 & SO/CPB/PB & 8 & SW & 0,50 & 521339 & 9747390 & 26599 \\
ANC1912 & 2250 & ST/AT & 30 & BM & 5,00 & 521413 & 9747223 & 60247 \\
Promedio & 2976 & & & & & & & 59634 \\
\hline
\end{tabular}

\section{Ubicación y geología del área}

En el área del pozo ANC1968 considerado se encuentran presentes las formaciones del Grupo Ancón (SO, CPB, ST) y la formación Atlanta correspondiente al Grupo Azúcar. Los pozos vecinos al pozo ANC1968 son el ANC0796 y ANC1946 ambos productores de las formaciones Atlanta y Santo Tomás (ver Figura 2).
La Arenisca Santo Tomas es de edad Eoceno Medio y está constituida de una arenisca silícea y conglomerática, fuertemente cementada con pequeñas intercalaciones de lutitas. Es una formación altamente resistiva que van de $10-70 \mathrm{ohms} / \mathrm{m}$, con porosidades bajas de $11 \%$ de promedio (Pacifpetrol, 2002). 


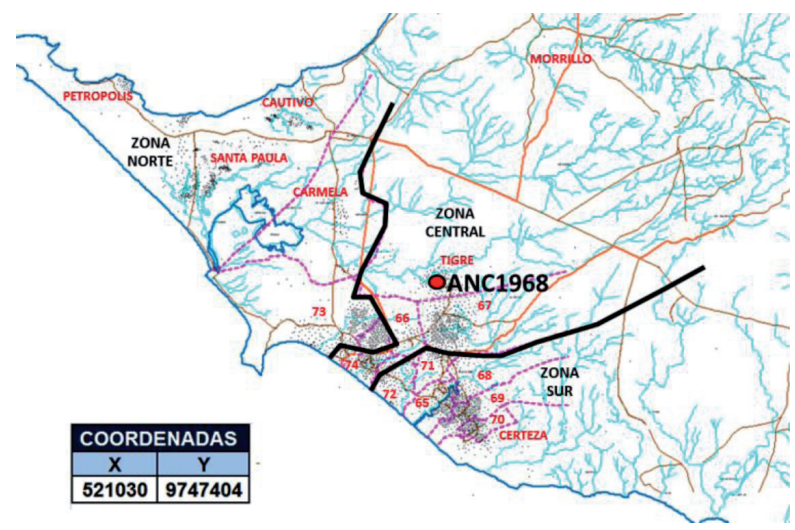

Figura 2. Mapa de ubicación ANC1968. Fuente: Pacifpetrol.

La Formación Atlanta de edad del Paleoceno-Eoceno Inferior, la cual descansa en discordancias sobre las rocas Cretácicas, está constituida de una arenisca gris dura, silícea con textura media a gruesa, con pequeñas intercalaciones de lutitas y conglomerados; representa el principal reservorio por sus fracturas (Pacifpetrol, 2002).

La correlación de los registros eléctricos de los pozos ANC1968, ANC1201 y ANC0796, sugiere la continuidad en la formación Santo Tomás en el área de estudio (Figura 3).

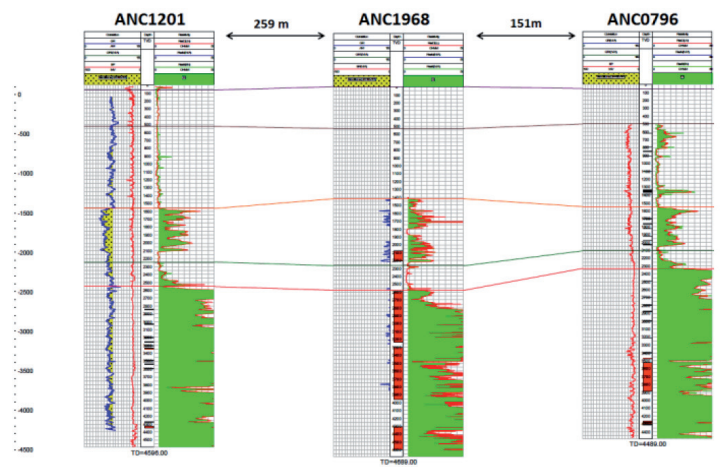

Figura 3. Sección geológica entre pozos ANC1201, ANC1968 y ANC0796. Fuente: Pacifpetrol.

\section{Historial de producción y reacondicionamientos}

El pozo ANC1968 entró en producción el 23-03-1966 con una producción inicial de 37 Bppd de la formación Atlanta que fue punzada con 58 shots y fracturada en el intervalo 3992'-4597'.

El 04-05-1966 el pozo fue punzado con 50 shots y fracturado en el intervalo 3299'-3960' de la formación Atlanta pasando su producción de 30 Bppd a 44 Bppd.
Posteriormente, el 13-07-1966 fue punzado con 43 shots y fracturado en el intervalo 2591'-3235' de la formación Atlanta incrementando su producción de 29 Bppd a 48 Bppd.

La producción declinó a 9 Bppd y el 07-04-1967 el pozo fue punzado con 20 shots y fracturado en el intervalo 2013'-2209' de la formación Santo Tomás obteniéndose una producción de 47 Bppd luego de la estimulación.

El pozo ha declinado su producción y actualmente se encuentra produciendo 2 Bppd con Bombeo Mecánico (Figura 4).

Considerando la curva de declinación, el pozo ANC1968 tiene reservas remanentes de unos 10,1 MBls (Pacifpetrol, 2015), como se puede constatar en la Figura 5.

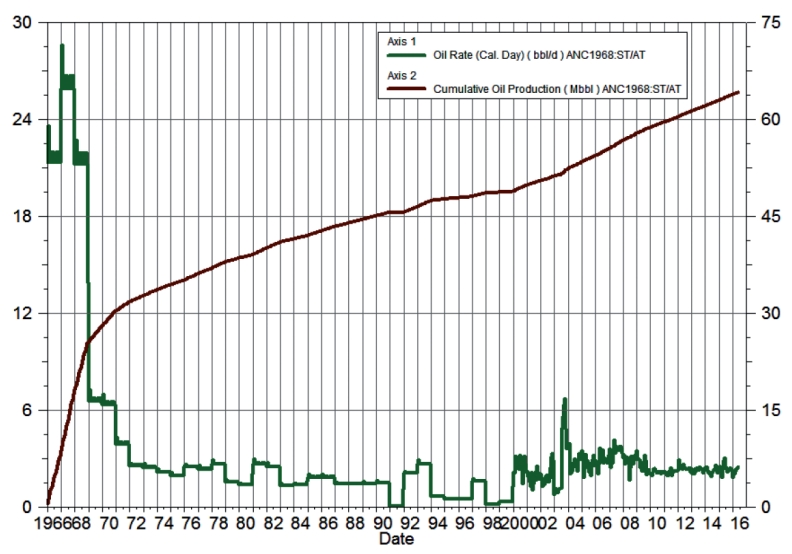

Figura 4. Historial de producción ANC1968. Fuente: Pacifpetrol.

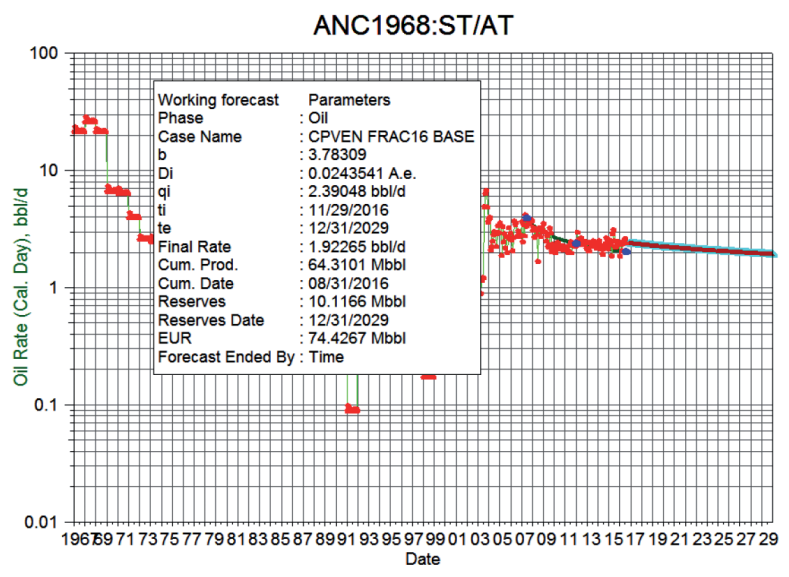

Figura 5. Declinación de producción ANC1968. Fuente: Pacifpetrol.

El pozo ANC1968 solo cuenta con los registros de Resistividad y Caliper. Para definir con exactitud los 
intervalos a ser punzados se deberá correr un Gamma Ray, sin embargo, por el registro de resistividad se puede visualizar que las zonas de interés están comprendidas entre 1630'-1720'.

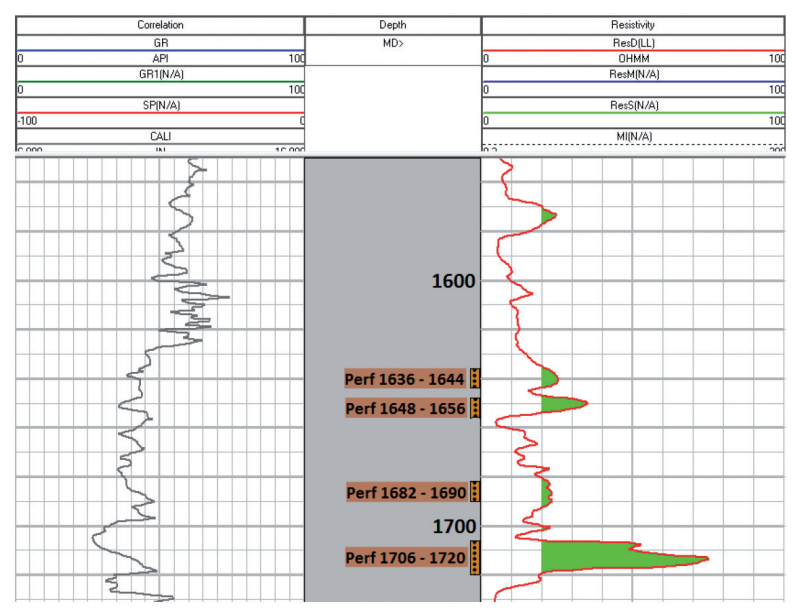

Figura 6. Registro ANC1968, Formación Santo Tomás. Fuente: Pacifpetrol.

\section{Cementación secundaria}

En abril 1967 se tomó un registro CBL en donde se observó que el tope de cemento se encontraba a +/-1960' razón por la cual, para realizar el trabajo de fractura propuesto, es necesario realizar una cementación secundaria (Squeeze).

Es importante mencionar que se recomienda correr un registro CBL/VDL a fin de verificar el tope del cemento y la adherencia cemento-formación-casing en la sección donde se realizará la fractura. Para un correcto desarrollo de la fractura (Medina, Martínez, León \& Boada, 2013), es necesario generar anillos de cemento (sello hidráulico) previo a realizar el trabajo de estimulación (Vázquez Cárdenas, 2009).

En el caso del pozo ANC1968 se ha considerado realizar dos trabajos de cementación secundaria mediante el método de Block Squeeze en los siguientes intervalos, los cuales serán disparados con cañones de $31 / 8$ " a 4 dpp (ver Tabla 2).

Tabla 2. Intervalos a punzar Squeeze ANC1968.

\begin{tabular}{|cccc|}
\hline Squeeze & Tope (ft) & Base (ft) & Intervalo (ft) \\
\hline 1 ero & 1770 & 1772 & 2 \\
\hline 2 do & 1620 & 1622 & 2 \\
Total & & & 4 \\
\hline
\end{tabular}

\section{Punzado y fracturamiento}

Del análisis de los registros eléctricos de los pozos, sus reservas, su comportamiento de producción y su reacción a las estimulaciones correspondientes para cada formación, se determinaron los intervalos de interés correspondientes a la parte superior de la formación Santo Tomás descritos en la Tabla 3 a ser disparados con cañones de $31 / 8$ " a 4 dpp.

Tabla 3. Intervalos a punzar ANC1968.

\begin{tabular}{|cccc|}
\hline Tope (ft) & Base (ft) & Intervalo (ft) & Cargas \\
\hline 1636 & 1644 & 8 & 32 \\
\hline 1648 & 1656 & 8 & 32 \\
\hline 1682 & 1690 & 8 & 32 \\
\hline 1706 & 1720 & 14 & 56 \\
\hline \multicolumn{2}{c}{ Total } & 38 & 152 \\
\hline
\end{tabular}

El intervalo a fracturar sería de $1636^{\prime}-1720^{\prime}$ de la formación Santo Tomás, con un volumen estimado de $516 \mathrm{Bls}$ de gel de fractura (SA-GELMAX-BC25\#) y gel lineal. Como material apuntalante (Serrano, Gómez, Rueda \& Núñez, 2013). Se recomienda inyectar 382 sacos de arena natural 12/20 (Figura 7).

Para realizar un adecuado seguimiento e interpretación de los resultados del plan de estimulación, se recomienda medir antes y después de la operación, el nivel de líquido en el pozo, el caudal de gas con las respectivas cromatografías de composición de gases, y la producción de petróleo y agua, con sus respectivos análisis de caracterización (Pazmiño Urquizo, 2004).

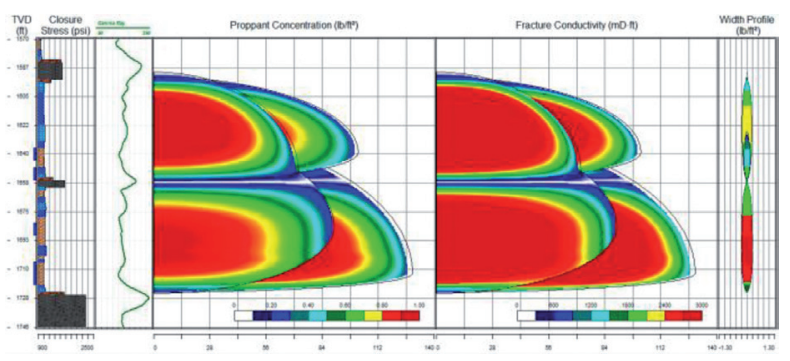

Figura 7. Diseño de fractura ANC1968. Fuente: CPVEN.

\section{Factibilidad económica}

Para la evaluación económica del proyecto de Fracturamiento Hidráulico en el Bloque Gustavo Galindo Velasco, se consideró como ejemplo las características del pozo ANC1968 y se evaluaron los costos, ingresos y se calculó el VAN y TIR (Baca, 2001), tomando en cuenta las siguientes consideraciones: 
El perfil de producción corresponde al incremental de producción debido a las fracturas realizadas en el pozo productivo de la sección Tigre, a partir de la ejecución del proyecto.

Se consideró una declinación hiperbólica del histórico de producción del mismo pozo tomando en cuenta que la zona propuesta a fracturar es la misma formación Santo Tomás. Además, se consideró una producción inicial de 12 BPPD después de la fractura (Figura 8).

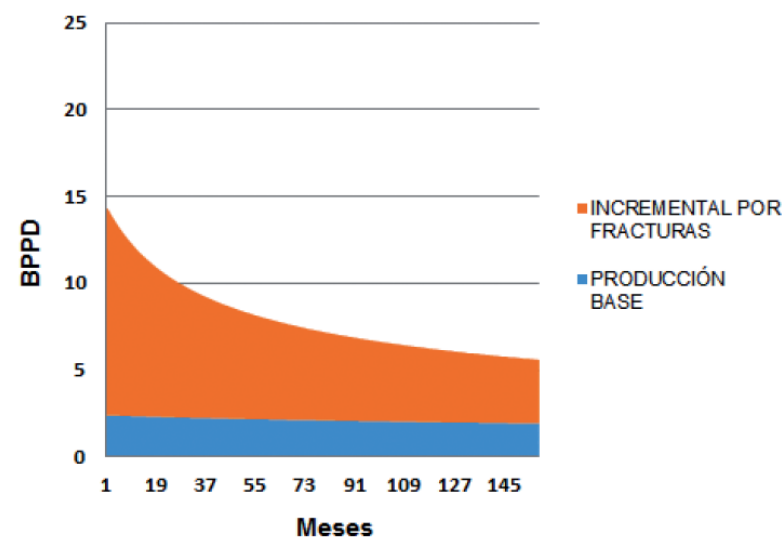

Figura 8. Perfil de producción ANC1968. Fuente: Pacifpetrol.

El ingreso disponible según contrato corresponde al $75 \%$ del precio WTI, mismo que es pagado por cada barril producido.

Los pozos fracturados producirán mediante Bombeo Mecánico, cuyo costo de levantamiento es de US\$1,25 por barril. Este costo está actualizado anualmente a una tasa de inflación del 4\%.

La inversión estimada requerida para el trabajo de fracturamiento es de US\$ 131.120, como se puede observar en la Tabla 4 que presenta los costos desglosados.
Tabla 4. Costos estimados ANC1968.

\begin{tabular}{lr}
\multicolumn{1}{|c}{$\begin{array}{c}\text { Fracturamiento Hidráulico } \\
\text { ANC1968 }\end{array}$} & \multicolumn{1}{c|}{$\begin{array}{c}\text { Costos } \\
\text { (\$) }\end{array}$} \\
\hline Toma de Registro GR-CCL-CBL-VDL & 8.500 \\
\hline Cementación Forzada & 25.000 \\
Cañoneo Selectivo & 31.000 \\
\hline Tapón CIBP + Asentamiento & 5.000 \\
Packer y Tapones Recuperables & 4.700 \\
1 Etapa de Fractura & 45.000 \\
10\% Imprevistos & 11.920 \\
\multicolumn{1}{c}{ Total } & 131.120 \\
\hline \multicolumn{1}{c}{} \\
\hline
\end{tabular}

En el análisis económico realizado para el pozo ANC1968, se consideraron los siguientes perfiles económicos referentes al precio WTI para el año 2017:

Perfil Mínimo. Se consideró un precio WTI de US\$ 40.33 por barril, se obtuvo un VAN de $\$ 160.223,89$, según proyección realizada hasta el final del contrato, un TIR de 57\% y un periodo de recuperación de 16 meses.

Perfil Probable. Se consideró un precio WTI de US\$ 45.33 por barril, se obtuvo un VAN de $\$ 196.782,80$, según proyección realizada hasta el final del contrato, un TIR de $67 \%$ y un periodo de recuperación de 14 meses.

Perfil Máximo. Se consideró un precio WTI de US\$ 50.33 por barril, se obtuvo un VAN de $\$ 233.361,70$, según proyección realizada hasta el final del contrato, un TIR de 79\% y un periodo de recuperación de 12 meses.

De acuerdo con los resultados obtenidos, el proyecto cumple con los criterios de rentabilidad exigida para su implementación (ver Tabla 5).

El autor expresa sus agradecimientos a la Empresa Pacifpetrol S.A. y a la Universidad Estatal Península de Santa Elena UPSE por su constante apoyo durante el desarrollo de este trabajo.

Tabla 5. Resultados evaluación económica.

\begin{tabular}{ccccccc}
$\begin{array}{c}\text { Perfiles } \\
\text { Económicos }\end{array}$ & $\begin{array}{c}\text { Precio Barril WTI } \\
\mathbf{( \$ )}\end{array}$ & $\begin{array}{c}\text { Inversión } \\
\mathbf{( \$ )}\end{array}$ & $\begin{array}{c}\text { VAN } \\
\mathbf{( \$ )}\end{array}$ & TIR (\%) & RBC & $\begin{array}{c}\text { Período de Recuperación } \\
\text { (Meses) }\end{array}$ \\
\hline Mínimo & 40,33 & $131.120,00$ & $160.223,89$ & 57 & 3,54 & 16 \\
Probable & 45,33 & $131.120,00$ & $196.792,80$ & 67 & 4,21 & 14 \\
Máximo & 50,33 & $131.120,00$ & $233.361,70$ & 79 & 4,89 & 12 \\
\hline
\end{tabular}




\section{Conclusiones}

El estudio realizado indica que el Bloque Gustavo Galindo Velazco, es un excelente candidato para incrementar su producción a través de la aplicación de la técnica de fracturamiento hidráulico en pozos de baja productividad.

El respectivo análisis técnico-económico ratifica los excelentes resultados obtenidos con la implementación del fracturamiento hidráulico, permitiendo de esta manera continuar con el desarrollo de nuevos trabajos en otros pozos de este campo.

Debido a los excelentes resultados obtenidos en la campaña de fracturamiento hidráulico del Bloque Gustavo Galindo Velazco, se recomienda implementar esta técnica en otros campos maduros, como un método eficiente para incrementar la producción de dichos campos.

\section{Referencias}

1. Baca, G. (2001). Evaluación de Proyectos. México D.F., México: McGraw-Hill.

2. Ecuador. Pacifpetrol. (2002). Informe Geológico y de Reservorios del Área. Ancón.

3. Ecuador. Pacifpetrol. (2015). Informe de Reservas del Bloque Gustavo Galindo Velazco. Ancón.
4. Escobar Macualo, F. H. (2000). Fundamentos de Ingeniería en Yacimientos. Neiva, Colombia: Universidad Surcolombiana.

5. Medina, C. A. C., Martínez, J. J. S., León, E. A., \& Boada, W. M. (2013). Análisis reológico para predecir y mejorar el comportamiento hidráulico durante la perforación de un pozo. Fuentes: El reventón energético, 11(1), 5.

6. Pazmiño, J. (2004). Fundamentos de la Teoría del Fracturamiento Hidráulico. Quito, Ecuador: Petroecuador.

7. Quintero, Y. A., Triana, R. L., Jaimes, M. G., \& Torres, M. L. (2010). Optimización de diseños de fractura hidráulica aplicando estudios geomecánicos. Revista fuentes, 8(2).

8. Serrano, D. S., Gómez, C. A. P., Rueda, R. C., \& Núñez, R. D. C. (2013). Implementación de propantes ultralivianos en el fracturamiento hidráulico de pozos. Fuentes: El reventón energético, 11(1), 1.

9. Vázquez Cárdenas, J. (2009). Mejoramiento de la Conductividad en el Fracturamiento Hidráulico. México D.F., México: UNAM.

Recepción: 15 de enero de 2018

Aceptación: 14 de mayo de 2018 